\title{
Otimização da Localização de Estações Radiobase Baseada em Sistemas Imunológicos Artificiais
}

\author{
Djalma de Melo Carvalho Filho e Marcelo Sampaio de Alencar
}

\begin{abstract}
This article describes base station placement as a multi-objective problem (MOP). Base station placement and configuration envolves a large number of unknowns and constraints and heuristic algorithms seem to be a suitable alternative to solve MOPs. A new class of evolutionary algorithms, the socalled multi-objective optimisation algorithms based on artificial immune systems (MO-AIS) are the core of an innovative approach to base station placement, which is presented in this paper. Preliminary results followed by a thorough analysis are provided. Three network simulation environments are used in the tests.
\end{abstract}

Resumo-Este trabalho descreve a implantação de estações radiobase como um problema de otimização multiobjetivo (MOP). A determinação da localização e da configuração de ERBs envolve um número elevado de variáveis e restrições de projeto e os algoritmos heurísticos representam uma alternativa viável para a resolução de MOPs. Uma nova classe de algoritmos evolutivos, os algoritmos de otimização multiobjetivo baseados em sistemas imunológicos artificiais (MO-AIS), constituem a base de uma nova estratégia de otimização da localização de ERBs apresentada. Resultados preliminares de análise dos algoritmos são apresentados. Três diferentes ambientes de simulação são utilizados.

Palavras-Chave-Estação radiobase, otimização multiobjetivo, sistemas imunológicos artificiais.

Keywords-Base station, multi-objective optimisation, artificial immune systems.

\section{INTRODUÇÃO}

O problema de localização de estações radiobase é um problema de otimização multiobjetivo (MOP). Deseja-se escolher o melhor conjunto de locais ou sítios disponíveis para a instalação e satisfazer várias restrições: garantir o handoff entre células e ao mesmo tempo o nível mínimo de interferência, produzir a melhor cobertura e suprir a demanda com custo mínimo de implantação e manutenção, respeitar a legislação ambiental quanto ao nível de potência dos sinais emitidos e quanto aos locais em que a instalação de antenas é vetada e cumprir as normas das agências reguladoras dos serviços de comunicações. Além dos locais selecionados, deve-se ainda determinar o número de antenas instaladas bem como suas respectivas configurações (tipo de antena, azimute, inclinação, ganho, potência emitida, etc.). Estimativas de tráfego e estudos do ambiente de propagação para uma dada região são utilizados.

Os modelos já propostos na literatura procuram contrabalançar tempo computacional e nível de detalhamento

Djalma de Melo Carvalho Filho e Marcelo Sampaio de Alencar Universidade Federal de Campina Grande, Centro de Engenharia Elétrica e Informática, Campina Grande, Brasil, E-mails: djalmacarvalho@uol.com.br, malencar@iecom.org.br. Este trabalho tem o apoio tecnológico do Instituto de Estudos Avançados em Comunicações (IECOM). do ambiente de simulação. Quanto mais detalhado e realista o modelo, maior o tempo de processamento até a obtenção de resultados satisfatórios. Além de diferenças em relação ao nível de detalhamento ou complexidade, os modelos já propostos apresentam diferenças em relação: ao espaço de simulação, ao tipo de planejamento, ao número de objetivos e a quais objetivos são considerados na otimização, aos modelos de propagação usados para o cálculo de predição de perda de percurso e aos algoritmos de otimização empregados.

Vários algoritmos de otimização já foram propostos: simulated annealing [1]; algoritmo de busca local [2]; algoritmo evolutivo [3]; algoritmo genético [4], [5]; tabu search [6].

Os algoritmos de otimização multiobjetivo baseados em sistemas imunológicos artificiais (MO-AIS) constituem uma nova classe de algoritmos evolutivos. Os algoritmos MO-AIS utilizam como inspiração mecanismos presentes nos sistemas imunológicos humanos, tais como: a maturação da afinidade, o reconhecimento de antígenos e a edição de receptores por meio de uma biblioteca DNA [7].

Vários algoritmos de otimização multiobjetivo baseados unicamente em sistemas imunológicos artificiais já foram propostos [8], [9]: Algoritmo imunológico de otimização multiobjetivo com restrições (CMOIA); Algoritmo imunológico de otimização multiobjetivo (MISA) [10]; Sistema imunológico vetorial (VIS) [11]; Algoritmo de seleção clonal multiobjetivo (MOCSA) [12]. A maior vantagem desses algoritmos em relação a outras abordagens inteligentes é a possibilidade de realizar buscas locais e buscas globais simultaneamente [13].

\section{A otimizaÇÃo multiobjetivo}

O problema de otimização multiobjetivo ou problema de otimização de critérios múltiplos é formalmente descrito a seguir.

Deseja-se encontrar o vetor

$$
\mathbf{x}=\left[x_{1}, x_{2}, . ., x_{n}\right]^{T}, \mathbf{x} \in \Omega
$$

que minimiza ou maximiza as componentes do vetor funções objetivo

$$
f(\mathbf{x})=\left[f_{1}(\mathbf{x}), f_{2}(\mathbf{x}), \ldots, f_{k}(\mathbf{x})\right]^{T},
$$

sujeito a

$$
g_{i}(\mathbf{x}) \leq 0 \text { para } i=1, \ldots, m,
$$

e

$$
h_{j}(\mathbf{x})=0 \text { para } j=1, \ldots, p,
$$

em que $\mathrm{x}$ é o vetor de variáveis de decisão ou vetor de soluções, $m$ é o número de restrições de desigualdade, $p$ é 
o número de restrições de igualdade e $\Omega$ contém todos os valores possíveis de $\mathbf{x}$ que avaliam $f(\mathbf{x})$ (região de decisão). As Equações (3) e (4) descrevem as dependências entre as demais variáveis de decisão e parâmetros que fazem parte do problema.

No contexto de otimização multiobjetivo, o conceito de ótimo global é substituído por uma solução de compromisso, que procura balancear os demais objetivos e restrições conflitantes. Essa solução de compromisso não é única. $\mathrm{O}$ conjunto de soluções plausíveis (ou candidatas) é denominado conjunto de ótimos de Pareto e compõem a frente de Pareto. Com base em informações acerca da frente de Pareto, as decisões específicas para a resolução de um dado problema podem ser tomadas com exatidão. Os conceitos de ótimo de Pareto e frente Pareto são apresentados a seguir.

Definição 1: (Dominância Pareto) $\mathrm{O}$ vetor $\mathbf{u}=$ $\left[u_{1}, u_{2}, . ., u_{k}\right]^{T}$ domina o vetor $\mathbf{v}=\left[v_{1}, v_{2}, . ., v_{k}\right]^{T}(\mathbf{u} \preceq \mathbf{v})$ se somente se $\mathbf{u}$ é parcialmente menor que $\mathbf{v}$, ou seja, $\forall i \in\{1, \ldots, k\}, u_{i} \leq v_{i} \wedge \exists i \in\{1, \ldots, k\}: u_{i}<v_{i}$.

Definição 2: (Pareto ótimo) $\mathrm{O}$ vetor $\mathrm{x}^{*} \in \Omega$ é um Pareto ótimo se não existe um vetor $\mathbf{x}^{\prime} \in \Omega$ tal que $\mathbf{v}=f\left(\mathbf{x}^{\prime}\right)=$ $\left[f_{1}\left(\mathbf{x}^{\prime}\right), f_{2}\left(\mathbf{x}^{\prime}\right), \ldots, f_{k}\left(\mathbf{x}^{\prime}\right)\right]^{T}$ domina $\mathbf{u}=f\left(\mathbf{x}^{*}\right)=$ $\left[f_{1}\left(\mathbf{x}^{*}\right), f_{2}\left(\mathbf{x}^{*}\right), \ldots, f_{k}\left(\mathbf{x}^{*}\right)\right]^{T}$. Uma solução não-dominada é um Pareto ótimo.

Definição 3: (Conjunto de ótimos de Pareto) Seja um problema de otimização multiobjetivo dado por $f(\mathbf{x})=$ $\left[f_{1}(\mathbf{x}), f_{2}(\mathbf{x}), \ldots, f_{k}(\mathbf{x})\right]^{T}$, o conjunto de ótimos de Pareto $P^{*}$ é definido por

$$
P^{*}:=\left\{\mathbf{x} \in \Omega \mid \neg \exists \mathbf{x}^{\prime} \in \Omega \quad f\left(\mathbf{x}^{\prime}\right) \preceq f(\mathbf{x})\right\} .
$$

Quando um vetor de soluções $\mathbf{x}$ que representa um pareto ótimo é avaliado por meio das funções objetivo $f(\mathbf{x})$, o vetor $\mathbf{u}$ é obtido. As componentes do vetor $\mathbf{u}$ representam os valores ótimos para cada um dos objetivos de otimização. Esses valores não podem ser melhorados isoladamente sem que os demais objetivos de otimização sejam afetados. O conjunto de todos os vetores de soluções que satisfazem as condições de optimalidade de Pareto formam o conjunto de ótimos de Pareto $P^{*}$.

Definição 4: (Frente de Pareto) Seja um problema de otimização multiobjetivo (MOP) dado por $f(\mathbf{x})=$ $\left[f_{1}(\mathbf{x}), f_{2}(\mathbf{x}), \ldots, f_{k}(\mathbf{x})\right]^{T}$ e pelo conjunto de ótimos de Pareto $P^{*}$, a frente de Pareto $P F^{*}$ é definida por

$$
P F^{*}:=\left\{\mathbf{u}=f(\mathbf{x}) \mid \mathbf{x} \in P^{*}\right\} .
$$

A frente de Pareto $P F^{*}$ é composta por todos os vetores obtidos ao se avaliar cada uma das soluções presentes no conjunto de ótimos de Pareto $P^{*}$. Todos os vetores que compõem a frente de Pareto são não-dominados. Como a determinação da frente de Pareto analiticamente nem sempre é possível para problemas de engenharia reais, aproximações são adotadas. Faz-se necessário a distinção entre a frente de Pareto real $P F_{r}^{*}$ e a frente de Pareto aproximada $P F_{a}^{*}$ resultante do procedimento de otimização.

\section{A. Os algoritmos de otimização multiobjetivo baseados em sistemas imunológicos artificiais}

A teoria de seleção clonal ou teoria de expansão clonal é o conceito mais utilizado no desenvolvimento de algoritmos de otimização multiobjetivo baseados em sistemas imunológicos artificiais (SIAs) [13], [10], [12]. O algoritmo de seleção clonal é inspirado no processo de expansão clonal que ocorre com as células B. No caso de células B, a expansão clonal envolve adaptação por meio de mutação (hipermutação somática) e inclui um mecanismo de seleção. Este mecanismo de seleção garante que apenas as células B capazes de gerar anticorpos com maior afinidade sobrevivam e possam posteriormente se transformar em células de memória. Esse procedimento conjunto de mutação e seleção é denominado maturação da afinidade da resposta imune.

Além da teoria de seleção clonal, os sistemas imunológicos artificiais para resolução de problemas de otimização multiobjetivo já propostos utilizam como inspiração outros mecanismos dos sistemas imunológicos, tais como [12]: a teoria da rede imunológica; edição de receptores por meio de uma biblioteca de DNA; linfocinas ou mensagens químicas.

Os algoritmos MO-AIS possuem em comum: a existência de uma população memória ou população offline, a avaliação das funções objetivos por meio dos conceitos de avidade e afinidade, um mecanismo de seleção para a clonagem das melhores soluções obtidas, uma etapa de proliferação e hipermutação somática e uma etapa de diversificação. As variáveis de decisão têm representação binária ou representação real.

A população offline também denominada população arquivo ou população memória armazena as melhores soluções obtidas, ou seja, a aproximação da frente de Pareto, considerando as relações de dominância entre os demais vetores resultantes do processo iterativo.

Em otimização, a afinidade é obtida por meio da avaliação da função objetivo que se deseja otimizar $f(\mathbf{x})$ e as demais restrições do problema (3) e (4). A avidade representa a intensidade da ligação total entre um antígeno $f(\mathbf{x})$ e um anticorpo (vetor solução $\mathbf{x}$ ), portanto, mede a qualidade da solução candidata. Em MOCSA, as soluções candidatas (anticorpos) são classificadas em frentes não-dominadas sucessivas com base nas relações de dominância.

A seleção para clonagem das melhores $N_{c}$ soluções pode ser realizada de maneira determinística ou estocástica. Para implementar o processo de seleção proporcional à afinidade entre anticorpos e antígenos ou seleção de acordo com a avidade, qualquer mecanismo de seleção comumente empregado em algoritmos evolutivos pode ser escolhido, tais como: seleção por roleta, seleção elitista, seleção por hierarquias, seleção por torneio e seleção bi-classista.

A taxa de mutação inversamente proporcional à afinidade e o número de clones proporcional à afinidade quando combinados conferem ao algoritmo um balanço entre mecanismo de busca local e mecanismo de busca global. Uma vez determinada a porcentagem de indivíduos selecionados para a 
clonagem, o número de clones gerados pode ser determinado de várias maneiras. O número de clones fixo e igual para cada anticorpo selecionado é bastante utilizado [7]. Quanto maior a quantidade de indivíduos proliferando, maior será o tempo de processamento. Em MOCSA, os anticorpos são clonados de acordo com a frente de Pareto a qual pertencem [12].

A diversificação nos algoritmos MO-AIS está relacionada ao mecanismo de busca global. Não está presente em todos os algoritmos. A diversidade é geralmente implementada ao se adicionar novas soluções aleatoriamente. Com base na teoria da rede imunológica, o operador de supressão é freqüentemente usado [11]. Quando dois anticorpos estão muito próximos, um deles pode reconhecer o outro e, por esta razão, um deles deve ser eliminado. Quando a distância euclidiana entre os dois anticorpos no espaço dos objetivos é maior que um valor referencial $\epsilon_{1}$, o anticorpo com maior afinidade é eliminado.

Em MOCSA, Campelo et al. aplicam a supressão tanto no espaço das variáveis de decisão, como no espaço dos objetivos [9]. Em uma versão mais recente de MOCSA, Campelo et al. aplicam a supressão apenas no espaço de objetivos [12]. Os vetores do espaço de objetivos são inicialmente normalizados ao hipercubo unitário para eliminar diferenças relativas entre os limites inferior e superior para cada objetivo. As distâncias entre os demais anticorpos $N$ da população memória são então calculadas. As distâncias entre cada indivíduo e os $k$ vizinhos mais próximos são obtidas, em que $k$ é dado por

$$
k=\operatorname{round}(\sqrt{N}) \text {. }
$$

O indíviduo com a menor soma das $k$ distâncias é eliminado, pois se encontra em uma região densa da frente de Pareto. $\mathrm{O}$ procedimento é repetido até a população memória atingir o tamanho definido pelo usuário.

\section{DESCRIÇÃO DO MODELO}

O modelo proposto usa pontos de teste discretos baseado em [5]. Os modelos de pontos de teste discretos simplificam a medição dos objetivos de otimização, permitem a liberdade na distribuição de sítios candidatos e são facilmente adaptados tanto ao planejamento inicial como à expansão de redes. A área de trabalho ou espaço de simulação W é discretizada em pontos de teste de coordenadas cartesianas $(x, y, z)$ a uma dada resolução. Os seguintes dados são definidos:

- Os pontos de teste de recepção RTP, nos quais a qualidade de sinal de recepção é medida;

- Os pontos de teste de serviço STP, nos quais o nível de sinal recebido deve estar acima do valor limite $S_{q}$, que assegura a qualidade do serviço;

- Os pontos de teste de tráfego TTP, que representam a demanda de tráfego medido em erlang;

- Os sítios candidatos ERB, que podem conter até 3 antenas (1 antena omnidirecional ou até 3 antenas direcionais);

- A matriz de anglo de incidência AIM, que define os ângulos verticais de cada ERB a cada RTP;

- A matriz de perda de percurso PLM, com informações de perda de percurso entre cada ERB e os demais pontos de teste de recepção RTP.
No cálculo de perdas de percurso é utilizada a fórmula empírica urbana padrão de Hata. O modelo considera ainda as perdas aleatórias de sombreamento como o modelo de Huang et al [4]. Essas perdas incluem variações com o tempo e podem tanto amplificar como atenuar o sinal de recepção. São obtidas do próximo valor pseudo-aleatório gaussiano $(\mu, \sigma)$, em que $\mu$ é o valor da perda de percurso e $\sigma$ é $4 \mathrm{~dB}$.

O modelo de alocação escolhido é de alocação pelo melhor servidor em que cada STP é servido pela ERB que emite o sinal de maior intensidade. A célula corresponde ao conjunto de STPs cobertos em que $P_{r} \geq-90 \mathrm{dBm}$ e dependendo do número de antenas instaladas cada sítio pode conter até três células.

O conjunto ERB' correponde ao conjunto de sítios ERB com pelo menos uma antena ativa que satisfazem os seguintes objetivos de otimização:

- Cobertura - É a soma de todos os $S T P_{i}$ cobertos na área de trabalho dividida pelo número total de STP em porcentagem. Logo,

$$
\mathrm{COVER}_{E R B^{\prime}}=\frac{\sum_{i=1}^{n_{S T P}} S T P_{i}}{n_{S T P}} \times 100,
$$

em que,

$$
S T P_{i}=\left\{\begin{array}{l}
1, \text { se } S T P_{i} \text { está coberto, } \\
0, \text { caso contrário; }
\end{array}\right.
$$

- Custo unitário - É o número de sítios com pelo menos uma antena ativa. Logo,

$$
\mathrm{CUSTO}_{E R B^{\prime}}=\sum_{E R B_{i} \in E R B^{\prime}} E R B_{i} ;
$$

- Capacidade de tráfego - É a soma do tráfego atual na rede dividido pela demanda de tráfego total e expresso em porcentagem. Logo,

$$
\operatorname{TRAF}_{E R B^{\prime}}=\frac{\sum_{i=1}^{n_{E R B^{\prime}}} T_{E R B_{i}^{\prime}}}{\sum_{i=1}^{n_{T T P}} T T P_{i}} \times 100
$$

A estratégia de otimização é dividida em três etapas: préprocessamento, inicialização de sítios e processo iterativo. $\mathrm{Na}$ etapa de pré-processamento, a configuração das antenas que garante que o tráfego máximo em cada célula não ultrapasse 5,4 erlang e o conjunto de STPs cobertos por cada sítio são determinados. A idéia principal do procedimento é dimensionar células uma única vez e considerar os demais objetivos no processo iterativo. Se todos os STPs estiverem cobertos, os TTPs também estarão, visto que $T T P \subseteq S T P \subseteq R T P$.

A inicialização de sítios com representação binária tem o objetivo de acelerar o processo iterativo e reduzir o esforço computacional [5]. Cada indivíduo de uma população é identificado por uma cadeia binária de comprimento igual ao número de sítios candidatos $n_{E R B}$. Sítios inativos e ativos são identificados, respectivamente, por 0 e 1 . A população inicial é gerada aleatoriamente e o número de sítios ativos é escolhido segundo o seguinte critério: Para o primeiro terço da população, o número de sítios ativos é escolhido entre 1 e o 
mínimo número de sítios capaz de satisfazer a demanda total de tráfego; Para o segundo terço da população, o número de sítios ativos é escolhido entre este mínimo número de sítios e duas vezes este valor.

O algoritmo utilizado no processo iterativo é baseado em MOCSA, inicialmente proposto por Campelo et al. [12]. MOCSA adota representação real de variáveis e se baseia no princípio de seleção clonal e na teoria de rede imunológica. MOCSA apresentou resultados satisfatórios em diversas aplicações quando comparados a outros algoritmos MO-AIS. O algoritmo multiobjetivo com representação binária BRMOA adota a representação binária para a representação de variáveis e a mutação gaussiana ou indutiva é substituída pela mutação uniforme [7], com probabilidade

$$
p_{m}=\frac{1}{\sqrt{n_{E R B}}} .
$$

\section{AnÁlise de Resultados}

Três diferentes ambientes de simulação foram utilizados para a análise e foram propostos em [5]. O ambiente de simulação 1 reproduz uma grande metrópole com o tráfego distribuído em função da distância em relação ao centro. Quanto mais próximo ao centro, o tráfego é maior e, consequientemente, é maior a dificuldade em satisfazer essa demanda. $\mathrm{O}$ ambiente de simulação 2 reproduz o tráfego distribuído ao longo de uma estrada hipotética em que cada TTP tem o mesmo valor de tráfego ( 0,75 erlang). $\mathrm{O}$ ambiente de simulação 3 combina os ambientes anteriores com aproximadamente metade do tráfego proveniente de cada situação. Além de combinar os dois modelos, o ambiente de simulação 3 tem um acréscimo no tráfego de aproximadamente $8 \%$. As Figuras 1, 2 e 3 mostram, respectivamente, o ambiente de simulação 1 , o ambiente de simulação 2 e o ambiente de simulação 3. A Tabela I mostra os dados dos três ambientes de simulação.

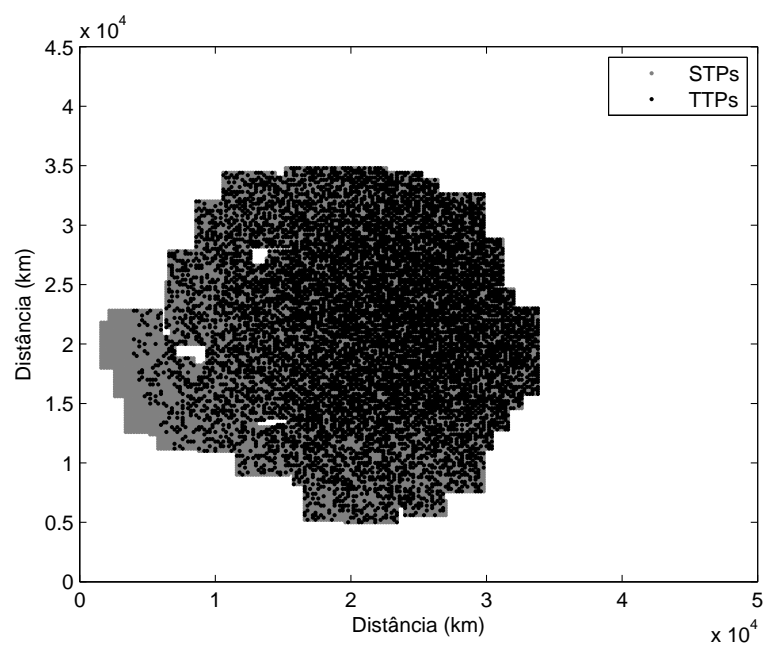

Fig. 1. Ambiente de simulação 1.

Os parâmetros de entrada de BRMOA definidos pelo usuário são: o tamanho da população inicial $P_{0}$, o número de gerações

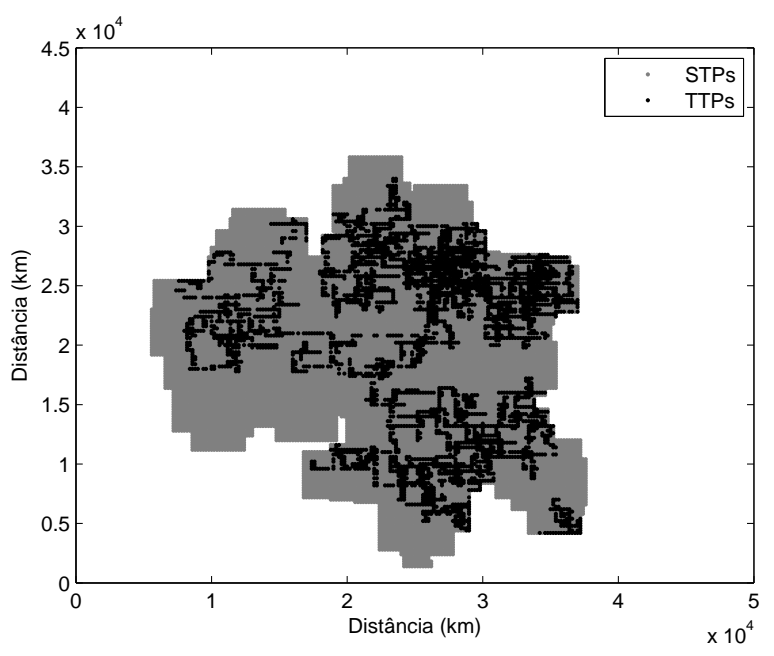

Fig. 2. Ambiente de simulação 2 .

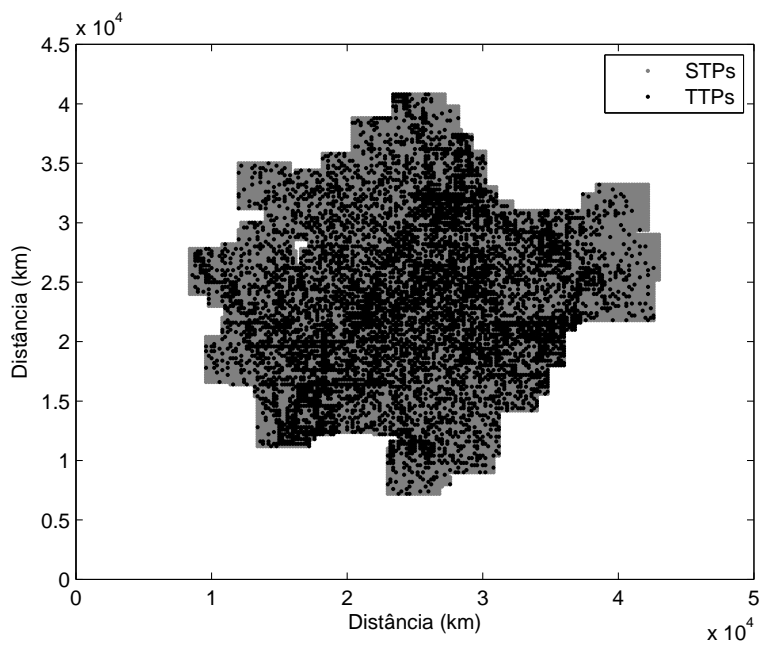

Fig. 3. Ambiente de simulação 3.

TABELA I

DADOS DO AMBIENTE DE SIMULAÇÃO.

$\begin{array}{cc}\text { Dimensões do terreno } & 2252,64 \mathrm{~m}^{2} \\ \text { Número de RTPs } & 56792 \\ \text { Número de STPs } & 17393 \\ \text { Número de sítios candidatos } & 568 \\ \text { Número de TTPs (ambiente 1) } & 8590 \\ \text { Número de TTPs (ambiente 2) } & 3985 \\ \text { Número de TTPs (ambiente 3) } & 6602 \\ \text { Demanda de tráfego total (ambiente 1) } & 2988,27 \text { erlang } \\ \text { Demanda de tráfego total (ambiente 2) } & 2988,75 \text { erlang } \\ \text { Demanda de tráfego total (ambiente 3) } & 3221,84 \text { erlang }\end{array}$


$g_{\max }$, o número máximo de avaliações da função objetivo $n_{e}$, o tamanho da população memória $P_{m}$ e a porcentagem de indivíduos substituídos a cada geração $d$.

Em problemas de otimização de larga escala como a otimização da localização de ERBs que envolve um número elevado de variáveis, o tempo de processamento bem como a qualidade das soluções obtidas têm importância fundamental. Em BRMOA é mantido um registro de todos os indivíduos já avaliados. Desta maneira, o número de avaliações da função objetivo durante o processo iterativo é reduzido. Para a análise das aproximações da frente de Pareto obtidas, vários indicadores de qualidade já foram sugeridos [9].

As aproximações da frente de Pareto foram obtidas para populações iniciais de 10,15 e 30 indivíduos, quando são considerados: $g_{\max }=10, n_{e}=12000, P_{m}=100$ e $d=0,25$. A análise estatística foi feita com base nos resultados obtidos em cinco execuções do programa, visto que o algoritmo BRMOA é um otimizador estocástico. A medida de dispersão de Schott foi escolhida como indicador de qualidade [9]. A medida de dispersão representa a variância da distância de cada elemento do conjunto de ótimos de Pareto obtidos em relação ao seu vizinho mais próximo e é dada por

$$
\begin{gathered}
S_{p}=\sqrt{\frac{1}{N-1} \sum_{i=1}^{N}\left(\bar{d}-d_{i}\right)^{2}}, \\
d_{i}=\min _{j}\left|f_{1}^{i}(\vec{x})-f_{1}^{j}(\vec{x})\right|+\left|f_{2}^{i}(\vec{x})-f_{2}^{j}(\vec{x})\right|,
\end{gathered}
$$

em que:

$N$ é o número de elementos que compõem a frente de Pareto $P F^{*}$;

$\bar{d}$ é o valor médio de todas as distâncias $d_{i}$;

$f_{k}^{i}(\vec{x})$ é cada uma das componentes da função objetivo do problema.

As Figuras 4, 5 e 6 mostram as melhores aproximações da frente de Pareto segundo a medida de dispersão de Schott para cada um dos ambientes de simulação. A Tabela II mostra a dispersão entre os valores obtidos para o ambiente de simulação 1. A Tabela III mostra a dispersão entre os valores obtidos para o ambiente de simulação 2. A Tabela IV mostra a dispersão entre os valores obtidos para o ambiente de simulação 3 .

\section{TABELA II}

DISPERSÃO ENTRE OS VALORES OBTIDOS EM CINCO EXECUÇÕES PARA CADA UMA DAS POPULAÇÕES INICIAIS PARA O AMBIENTE 1.

$\begin{array}{cccc}\text { Dispersão } & 10 \text { indivíduos } & 15 \text { indivíduos } & 30 \text { indivíduos } \\ \text { média } & 6,4214 & 2,6391 & 2,3773 \\ \text { mínima } & 2,9328 & 1,6129 & 2,2339 \\ \text { máxima } & 8,213 & 3,6652 & 2,6387 \\ \text { desvio padrão } & 2,1329 & 1,4512 & 0,1794\end{array}$

Com base nos resultados obtidos, é possível afirmar que uma aproximação razoável da frente de Pareto segundo o critério de dispersão de Schott pode ser obtida com uma população inicial de 15 indivíduos e após 459 avaliações do vetor funções objetivo para o ambiente de simulação 1, 469 avaliações para o ambiente 2 e 463 avaliações para o

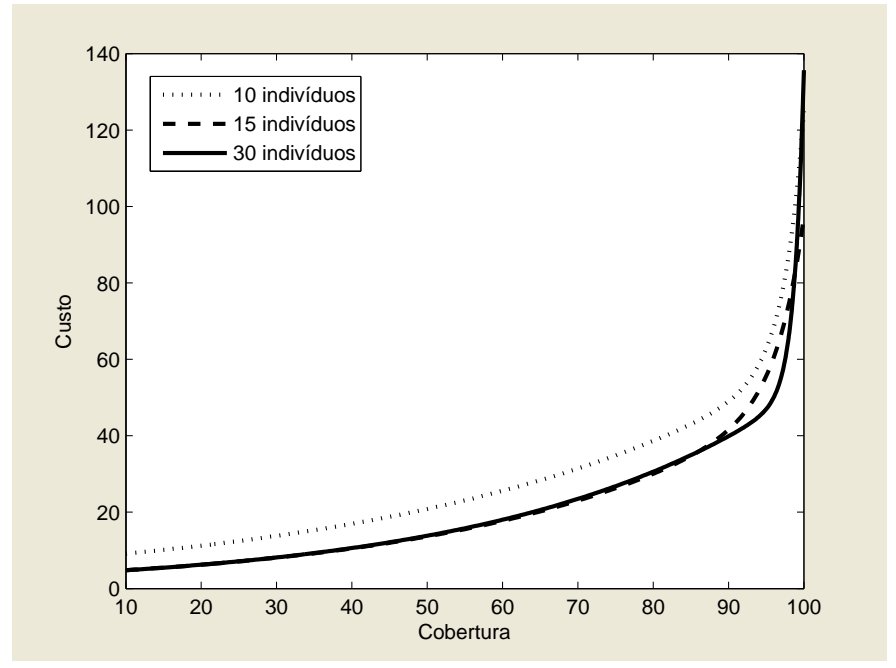

Fig. 4. Aproximação de Pareto para diferentes populações iniciais para o ambiente de simulação 1 .

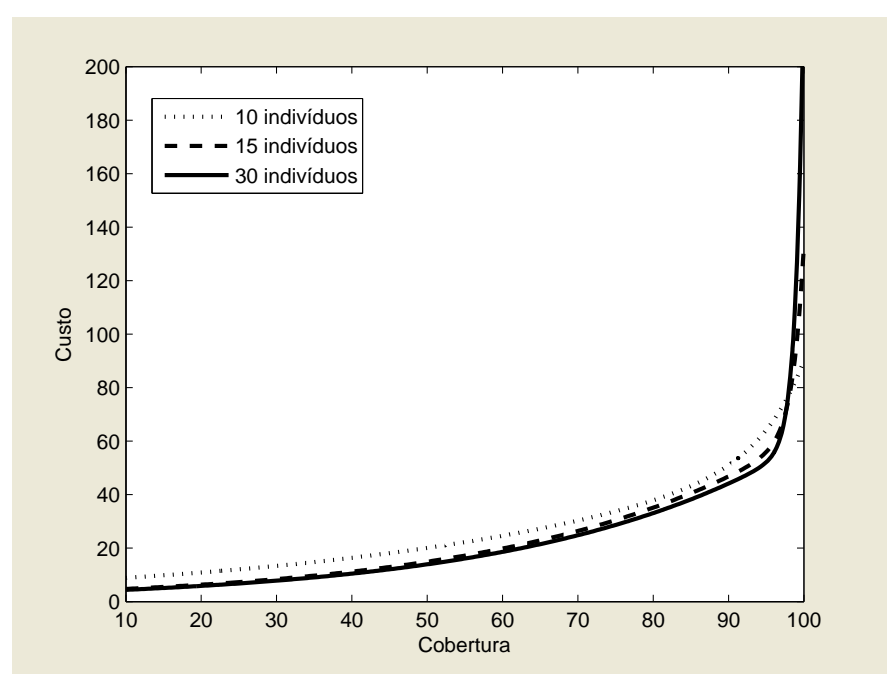

Fig. 5. Aproximação de Pareto para diferentes populações iniciais para o ambiente de simulação 2 .

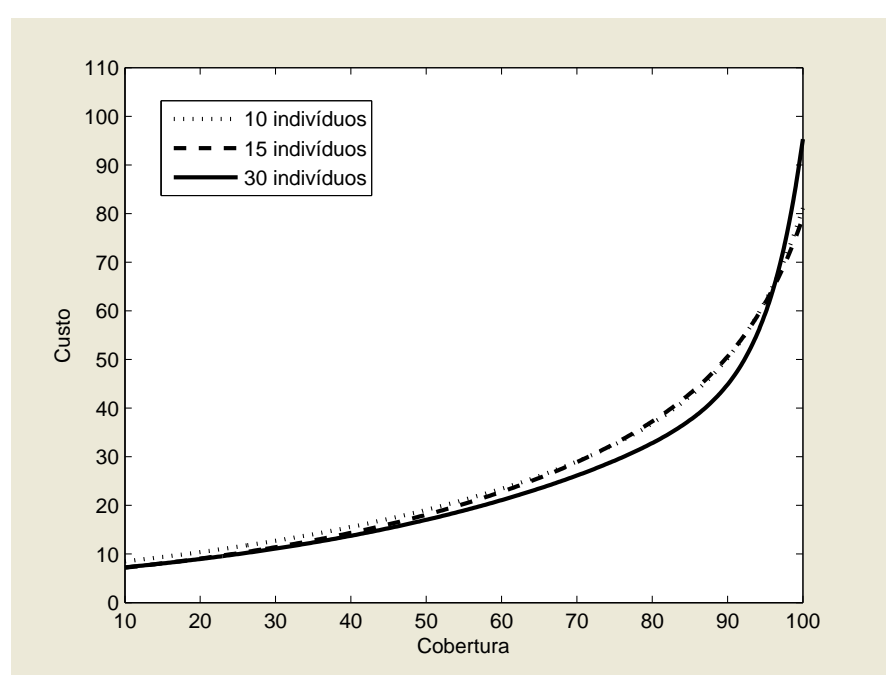

Fig. 6. Aproximação de Pareto para diferentes populações iniciais para o ambiente de simulação 3 . 
TABELA III

DISPERSÃO ENTRE OS VALORES OBTIDOS EM CINCO EXECUÇÕES PARA CADA UMA DAS POPULAÇÕES INICIAIS PARA O AMBIENTE 2.

$\begin{array}{cccc}\text { Dispersão } & 10 \text { indivíduos } & 15 \text { indivíduos } & 30 \text { indivíduos } \\ \text { média } & 4,1834 & 3,2324 & 3,2206 \\ \text { mínima } & 3,7815 & 0,9539 & 2,5685 \\ \text { máxima } & 4,9320 & 4,5818 & 4,5745 \\ \text { desvio padrão } & 0,6489 & 1,9844 & 0,9360\end{array}$

TABELA IV

DISPERSÃO ENTRE OS VALORES OBTIDOS EM CINCO EXECUÇÕES PARA CADA UMA DAS POPULAÇÕES INICIAIS PARA O AMBIENTE 3.

$\begin{array}{cccc}\text { Dispersão } & 10 \text { indivíduos } & 15 \text { indivíduos } & 30 \text { indivíduos } \\ \text { média } & 2,7272 & 2,2032 & 2,1828 \\ \text { mínima } & 2,2015 & 2,0349 & 1,7329 \\ \text { máxima } & 3,2528 & 2,3067 & 2,8453 \\ \text { desvio padrão } & 0,7434 & 0,1470 & 0,1164\end{array}$

ambiente 3. Raisanen et al. obtiveram resultados semelhantes por meio de um algoritmo genético ao considerar uma população inicial de 800 indivíduos, ou seja, 800 avaliações iniciais da função objetivo [5]. As melhores aproximações são obtidas quando uma população inicial de 30 indivíduos é considerada e após 3000 avaliações do vetor funções objetivo. Os resultados mostram ainda as soluções bem distribuídas ao longo da frente de Pareto, mesmo quando são consideradas populações iniciais pequenas. Para o ambiente de simulação 3, uma população inicial de apenas 10 indivíduos é capaz de produzir resultados satisfatórios. Essa característica dos algoritmos MO-AIS viabiliza o seu uso como otimizadores multiobjetivos.

Os custos de implantação de ERBs são bastante elevados para garantir $100 \%$ de cobertura. O ambiente de simulação 2 apresenta o maior custo de implantação para cobertura total, enquanto que o ambiente 3 tem o menor custo. Os custos são razoáveis para cobertura de 90-97\%. Para uma cobertura de $95 \%$, o ambiente de simulação 3 apresenta maior custo de implantação e, portanto, maior desafio na escolha da melhor configuração para a rede. Por outro lado, o ambiente de simulação 1 representa a melhor relação custo-benefício. A Figura 7 mostra as aproximações da frente de Pareto para diferentes gerações com população inicial de 30 indivíduos para o ambiente de simulação 1. Quando uma população inicial de 30 indivíduos é considerada, as aproximações obtidas nas primeiras gerações produzem resultados satisfatórios. Uma população inicial de 30 indivíduos é recomendável quando não há preocupação com o tempo de processamento.

Os resultados obtidos são compatíveis com os resultados apresentados na literatura recente e mostram que o uso de algoritmos MO-AIS para a otimização da localização de ERBs é uma alternativa promissora. Novos experimentos estão em curso no sentido de garantir uma solução de compromisso entre precisão e esforço computacional.

\section{CONCLUSÕES}

Este trabalho apresenta uma nova estratégia de otimização da localização de estações radiobase. Um algoritmo baseado

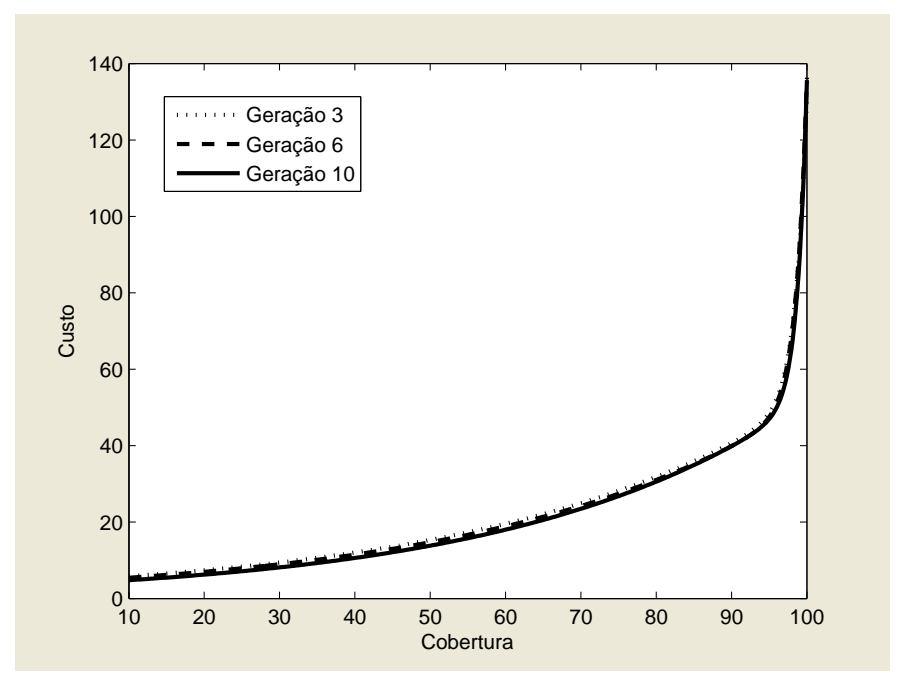

Fig. 7. Aproximação de Pareto para diferentes gerações com população inicial de 30 indivíduos para o ambiente de simulação 1 .

em sistemas imunológicos artificiais é utilizado como otimizador multiobjetivo. Três ambientes de simulação distintos são usados para avaliar o algoritmo. Resultados preliminares da análise do algoritmo MO-AIS são apresentados.

\section{REFERÊNCIAS}

[1] J. Ong C. Soh Q. Hao, B. Soong and Z. Li. A low-cost cellular mobile communication system: A hierarquical optimization network resource planning approach. IEEE Journal on Selected Areas in Communications, 15(7):1315-1326, September 1997.

[2] R. K. Rawnsley and S. Hurley. Towards automatic cell planning. In The 11th IEEE International Symposium on Personal, Indoor and Mobile Radio Communications, 2000 - PIMRC 2000, London, UK, pages 15831588, September 2000.

[3] R. K. Taplin S. M. Allen, S. Hurley and R. M. Whitaker. Automatic cell planning of broadband fixed wireless networks. In The 53rd IEEE Vehicular Technology Conference - VTC 2001 Spring, Rhodes, Greece, volume 4, pages 2808-2812, May 2001.

[4] U. Behr X. Huang and W. Wiesbeck. Automatic cell planning for a lowcost and spectrum efficient wireless network. In Global Telecommunications Conference, GLOBECOM '00, San Francisco, USA, November 2000 .

[5] L. Raisanen. A permutation-coded evolutionary strategy for multiobjective GSM network planning. Journal of Heuristics, 2007.

[6] M. Vasquez and J-K. Hao. A heuristic approach for antenna positioning in cellular networks. Journal of Heuristics, 7:443-472, 2001.

[7] Leandro N. de Castro and Jonathan Timmis. Artificial Immune Systems: A New Computational Intelligence Approach. Springer, 2002.

[8] F. Guimarães F. Campelo and H. Igarashi. Overview of artificial immune systems for multi-objective optimization. In Carlo Poloni Tomoyuki Hiroyasu Shigeru Obayashi, Kalyanmoy Deb and Tadahiko Murata, editors, 4th International Conference on Evolutionary MultiCriterion Optimization, EMO 2007, pages 937-951. Springer, March 2007.

[9] Gary B. Lamont Carlos A. Coello Coello and David A. Van Veldhuizen. Evolutionary Algorithms for Solving Multi-Objective Problems. Springer, 2007.

[10] Nareli Cruz Cortés and Carlos A. Coello Coello. Solving multiobjective optimization problems using an artificial immune system. Genetic Programming and Evolvable Machines, 6:163-190, 2005.

[11] F. Freschi. VIS: An artificial immune network for multi-objective optimization. Engineering optimization, 38(8):975-996, December 2006.

[12] F. Campelo F. G. Guimarães, R. M. Palhares and H. Igarashi. Design of mixed control systems using algorithms inspired by the immune system. Information Sciences, 177:4368-4386, 2007.

[13] G. Luh and C. Chueh. Multi-objective optimal design of truss structure with immune algorithm. Computers and Structures, 82:829-844, 2004. 